\section{Which Ras rides the raft?}

To the editor - Biochemical and morphological analysis of plasma membranes has identified coherent lipid domains called rafts or caveolae that dynamically organize multiple membrane proteins. Ras, Ras activators and Ras effectors are found in caveolae/rafts, indicating that they may be sites of Ras action. An unresolved question of Ras function is the relative contribution of each of the three Ras isoforms $(\mathrm{H}, \mathrm{K}$ and $\mathrm{N}$ ) to the myriad cellular responses attributed to activated Ras. The importance of this question is highlighted by the specificity of activation of distinct Ras isoforms in various classes of human tumours, and by the dramatic difference each isoform contributes to mouse development.

Recent work by Hancock and colleagues $^{1,2}$ has generated the intriguing hypothesis that functional differences between Ras isoforms are due, at least in part, to localization in different plasma membrane compartments: H-Ras to caveolae/rafts and K-Ras to an unidentified, non-caveolar membrane domain. The primary difference between the Ras isoforms is their carboxy-terminal membrane targeting sequence. Both $\mathrm{H}$ - and $\mathrm{K}$-Ras are prenylated but H-Ras is additionally modified by palmitoylation whereas K-Ras contains a basic, polylysine sequence.

Prior et al. suggest that the membrane anchors confer targeting specificity for distinct membrane domains ${ }^{1}$. Despite the attractiveness of a multi-compartment model to explain how Ras isoforms can have different functions $s^{3,4}$, it is important to consider a number of published observations that do not support this model. Prior et al. ${ }^{1}$ and Roy et al. ${ }^{2}$ used a $\mathrm{pH} 11$ carbonate buffer to prepare caveolar membranes. However, when caveolae are prepared from sonicated plasma membranes using Optiprep gradients at physiological $\mathrm{pH}$ and ionic strength, both endogenous $\mathrm{H}$-Ras and K-Ras are highly enriched in caveolae fractions ${ }^{5}$. Moreover, EGF stimulates recruitment to and activation of Raf exclusively in the caveolae fraction ${ }^{6}$. Disruption of caveolae/rafts by cholesterol depletion causes a loss of both K-Ras and $\mathrm{H}$-Ras from caveolae fractions, and recruitment of Raf to caveolae is impaired ${ }^{5}$, indicating that both $\mathrm{H}$ - and $\mathrm{K}$ Ras are in cholesterol-rich domains.

Native Ras has not been successfully immunolocalized in cells. Endogenous RhoA, however, a Ras superfamily member that is membrane anchored by a combination of a prenyl group and a polybasic domain similar to that found in K-Ras, has been successfully localized to caveolae by immunogold electron microscopy ${ }^{7}$.

Finally, there is compelling evidence that the entire Ras/Raf/Mek/Erk cascade is compartmentalized in caveolae/rafts and that Erk can be activated in purified caveolae fractions by $\mathrm{PDGF}^{8,9}$. These findings are more consistent with a model where both H-Ras and K-Ras carry out critical signalling functions in caveolae/rafts. Methodology can have a dramatic impact on the nature of whatever system is under study. Clearly, observations of protein localization to plasma membrane compartments are particularly sensitive to the techniques used to make the observations. Further efforts are needed to understand how Ras is compartmentalized, and the consequences of this on Ras function.

\section{Michael A. White ${ }^{*}$, Richard G. W. Anderson†}

${ }^{*}$ Department of Cell Biology and †Department of Cell Biology and Neuroscience, University of Texas Southwestern Medical Center, Dallas, Texas, USA

e-mail:michael.white@UTSouthwestern.edu; anders06@utsw.swmed.edu

Reply - We have used multiple techniques to address the issue of Ras localization and shown for the first time that $\mathrm{H}$ and $\mathrm{K}$-ras have different distributions across caveolae, raft and non-raft domains (as defined in ref. 10). Anderson and White, however, argue for the exclusive caveolar localization of Ras proteins based solely on their biochemical studies showing that H-Ras and K-Ras cofractionate in lowdensity membranes in what they refer to as a caveolae fraction. Many of their comments follow from the inadequate resolution of their fractionation procedure. Given that they find that two proteins cofractionate and yet we can resolve them (by whatever method), it is self-evident that they have poor separation. Also how pure is the Anderson lab's caveolae fraction? It is important to note that recent work ${ }^{11}$ questioned their caveolar-localization of EGF receptors. Careful inspection of the Optiprep method that they used ${ }^{6}$ raises additional questions. Although there is overlap between caveolin and H-Ras on their initial gradient, the distributions of the two proteins are definitely not concordant and look very similar to the relative distributions we observed ${ }^{1}$. Second, they discard a substantial number of Ras- and Raf-containing fractions and disordered membrane fractions before isolating a caveolae fraction. These technical deficiencies would make it impossible to observe the differential Ras localizations shown in Prior et al. ${ }^{1}$ because so much of the plasma membrane is excluded from analysis. Although we emphasize the role of rafts in H-Ras localization and function, we did find a small proportion of $\mathrm{K}$ Ras cofractionating with raft markers ${ }^{1}$. The significance of this is unknown.

Importantly, we correlated the raft/non-raft association of Ras, determined biochemically, with a detailed electron microscopy and functional analysis ${ }^{1}$. Our data are completely concordant with respect to: the different plasma membrane localizations of $\mathrm{H}$-Ras and $\mathrm{K}$-Ras (including endogenous $\mathrm{H}$-Ras and $\mathrm{K}$ Ras); the effect of GTP loading on H-Ras lateral segregation; and functional studies showing that H-Ras but not K-Ras signalling is cholesterol dependent ${ }^{2}$. The comments on Rho proteins emphasize another tenet of our paper: protein sequences adjacent to the membrane anchor have a profound influence over lipid raft association. These sequences are non-conserved between Rho and Ras so extrapolating from one protein to another is presently impossible. In addition, although certain components of the MAPK cascade have been found in caveolae, only our study provides quantitative electron microscopy data on the localization of Ras proteins, an essential technique for assessing caveolar localization.

\section{Ian A Prior*, Robert G Parton†, John F Hancock*}

${ }^{*}$ Laboratory of Experimental Oncology, Department of Pathology, University of Queensland Medical School, Herston Road, Queensland 4006,

Australia

†Institute for Molecular Bioscience, Centre for Microscopy and Microanalysis and Department of Physiology and Pharmacology, University of Queensland, Queensland 4072, Australia e-mail: j.hancock@mailbox.uq.edu.au

Prior, I. A. et al. Nature Cell Biol. 3, 368-375 (2001).

. Roy, S. et al. Nature Cell Biol. 1, 98-105 (1999).

Shields, J. M., Pruitt, K., McFall, A., Shaub, A. \& Der, C. J. Trends Cell Biol. 10, 147-154 (2000).

4. Reuther, G. W. \& Der, C. J. Curr Opin. Cell Biol. 12, 157-165 (2000).

5. Furuchi, T. \& Anderson, R. G. J. Biol. Chem. 273, 21099-21104 (1998).

6. Mineo, C., James, G. L., Smart, E. J. \& Anderson, R. G. W. J. Biol. Chem. 271, 11930-11935 (1996).

7. Michaely, P. A., Mineo, C., Ying, Y. S. \& Anderson, R. G. J. Biol. Chem. 274, 21430-21436 (1999).

8. Liu, P., Ying, Y. \& Anderson, R. G. Proc. Natl Acad. Sci. USA 94, 13666-13670 (1997).

9. Liu, P., Ying, Y., Ko, Y. G. \& Anderson, R. G. J. Biol. Chem. 271, 10299-10303 (1996).

10. Simons, K. \& Toomre, D. Nature Rev. Mol. Cell Biol. 1, 31-39 (2000).

11. Waugh, M., Lawson, D. \& Hsuan, J. Biochem. J. 337, 191-197 (1999). 